\title{
On the influence of spatial sampling on climate networks
}

\author{
N. Molkenthin ${ }^{1,2}$, K. Rehfeld ${ }^{1,3}$, V. Stolbova ${ }^{1,2}$, L. Tupikina ${ }^{1,2}$, and J. Kurths ${ }^{1,2}$ \\ ${ }^{1}$ PIK Potsdam Institute of Climate Impact Research, P.O. Box 601203, 14412 Potsdam, Germany \\ ${ }^{2}$ Department of Physics, Humboldt-Universität zu Berlin, Newtonstr. 15, 12489 Berlin, Germany \\ ${ }^{3}$ Alfred-Wegner Institute for Polar and Marine Research, Telegrafenberg A43, 14473 Potsdam, Germany
}

Correspondence to: N. Molkenthin (molkenthin@ @ik-potsdam.de)

Received: 17 December 2013 - Revised: 11 March 2014 - Accepted: 11 April 2014 - Published: 3 June 2014

\begin{abstract}
Climate networks are constructed from climate time series data using correlation measures. It is widely accepted that the geographical proximity, as well as other geographical features such as ocean and atmospheric currents, have a large impact on the observable time-series similarity. Therefore it is to be expected that the spatial sampling will influence the reconstructed network. Here we investigate this by comparing analytical flow networks, networks generated with the START model and networks from temperature data from the Asian monsoon domain. We evaluate them on a regular grid, a grid with added random jittering and two variations of clustered sampling. We find that the impact of the spatial sampling on most network measures only distorts the plots if the node distribution is significantly inhomogeneous. As a simple diagnostic measure for the detection of inhomogeneous sampling we suggest the Voronoi cell size distribution.
\end{abstract}

\section{Introduction}

Complex networks are used in many areas of physics to describe systems as different as the brain, the internet and social interactions (Boccaletti et al., 2006; Barthélemy, 2011). While the first applications were discrete by nature, complex networks have recently also found use in continuous systems like climate (Tsonis et al., 2006; Donges et al., 2009; Hlinka et al., 2013) . Additionally in climate networks, spatial embedding plays a big role. While the properties of some spatially embedded networks, like those discussed in Barthélemy (2011) and Kosmidis et al. (2008), have been analyzed, their construction mechanisms are very different from that of climate networks. In those applications, the system's spatial description is reduced to a number of discrete points, between which correlations are evaluated to construct a network (Malik et al., 2011; Rehfeld et al., 2012; Stolbova et al., 2014). This reveals a variety of interesting structures, many of which can be explained with geographical features. Spatial proximity often leads to large correlations, but mountains for example, can act as a barrier and prohibit exchange. Regions with a dominant wind or ocean flow can exhibit longer links. With all this, an effect of the sampling locations has to be expected.

Aliasing effects can occur if the sampling steps are larger than the half-width of investigated phenomena and can distort the actual signal substantially (Unser, 2000; Dippé and Wold, 1985). Spatial sampling effects have been discussed (Heitzig et al., 2012), where they proposed weighted measures to deal with differently sized nodes. In climate networks the related issue of temporal sampling and irregularity are treated in Rehfeld et al. (2011) and the role of boundary effects was investigated in Rheinwalt et al. (2012). While previous studies have mostly used grids for the locations of the measurements (Yamasaki et al., 2008; Tsonis et al., 2010), in this paper we study the effects of the spatial sampling itself on two typical model example systems and one set of observational data. In contrast to previous studies, we look at the sampling effects compared to the underlying system, rather than just differently sized regions. The first example is a model that generates networks directly from flows, introduced in Molkenthin et al. (2013). The second example is the START model, introduced in Rehfeld et al. (2013), in which artificial autocorrelated time series are transported by a flow field. In these models the node locations can be chosen freely, making them ideal for studying the effects of spatial sampling. The third example is the analysis of temperature data from the Asian monsoon area. 

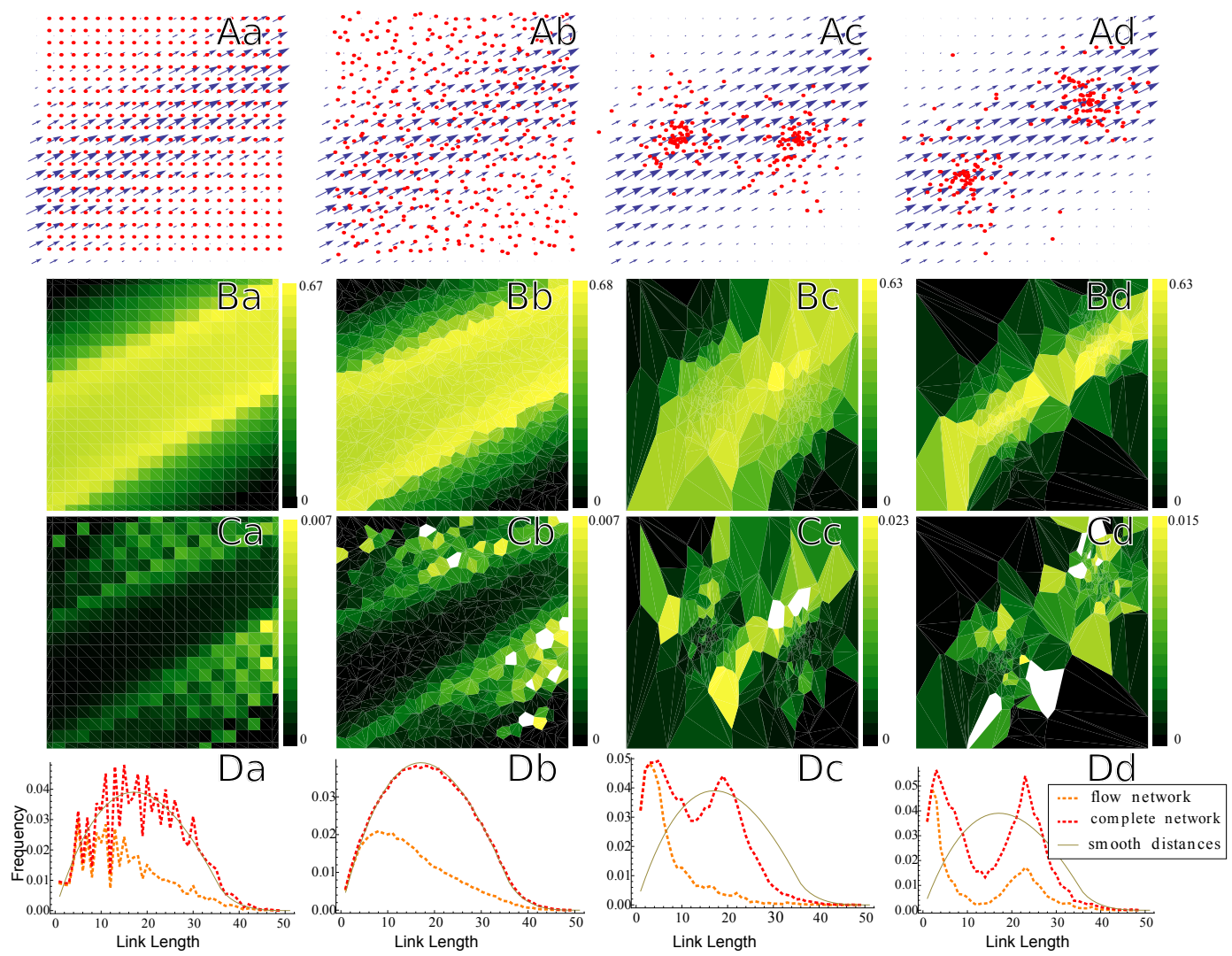

Figure 1. A diagonal flow is sampled with (Aa) a grid, (Ab) a jittered grid, (Ac) two clusters on opposite sides of the flow, and (Ad) two clusters inside the flow. The networks are constructed with a link density of $40 \%$. Row (B) shows the degree, row (C) the betweenness and row (D) the link length distribution.

We compute networks of a regular grid, a grid with a uniformly random jitter and clustered node distributions and compare their degree, betweenness and link length distribution to see how the spatial sampling affects common network measures.

\section{Spatial effects in flow networks}

Flow networks as introduced in Molkenthin et al. (2013) are constructed using analytical solutions of the advectiondiffusion-equation (ADE), instead of time series in the definition of the correlation. The correlation measure is based on the scalar product between the temperature profile of a single $\delta$-peak's evolution due to advection and diffusion, evaluated at each pair of nodes. We construct these networks and analyze the network measures degree, betweenness and link length distribution of the resulting networks and compare the influence of the spatial sampling on the result. Flow networks for a diagonal flow pattern are constructed for the node distributions shown in row A of Fig. 1, on a regular $20 \times 20$ grid, a jittered $20 \times 20$ grid and for two versions of Gaussian clustering. The jittered sampling pattern was generated by uniformly drawing a point from each grid cell. For the clustering, we used a Gaussian distribution around two central points on opposite sides of the flow (Ac) and along the flow (Ad) with 200 nodes. We plotted the node distribution in the flow, node degrees, node betweenness and the link length distribution for each of the sampling patterns.

Degree: we observe that the degree (shown in row B in Fig. 1) is higher in the middle stripe, where the flow velocity is highest. The degree is not very sensitive to the spatial sampling. In all four plots the degree is clearly highest where the absolute velocity is highest. Clustered sampling can lead to a skewed shape of the stripe, or underestimate its width if the outer region is poorly sampled.

Betweenness: the betweenness is shown in row C in Fig. 1. Grid and grid plus jitter show similar patterns: the betweenness is highest in the transition zone between the fast and slow flowing areas. This structure is barely visible in the clustered sampling plots. In the clusters on opposite sides, two stripes are visible, but the lower one is located in the center of the flow, rather than at its side, in the middle between the two clusters.

This indicates that rather than showing a transition zone of the flow, it emphasizes a region that is poorly sampled but central in the flow. In the plot of the two clusters in the flow, 


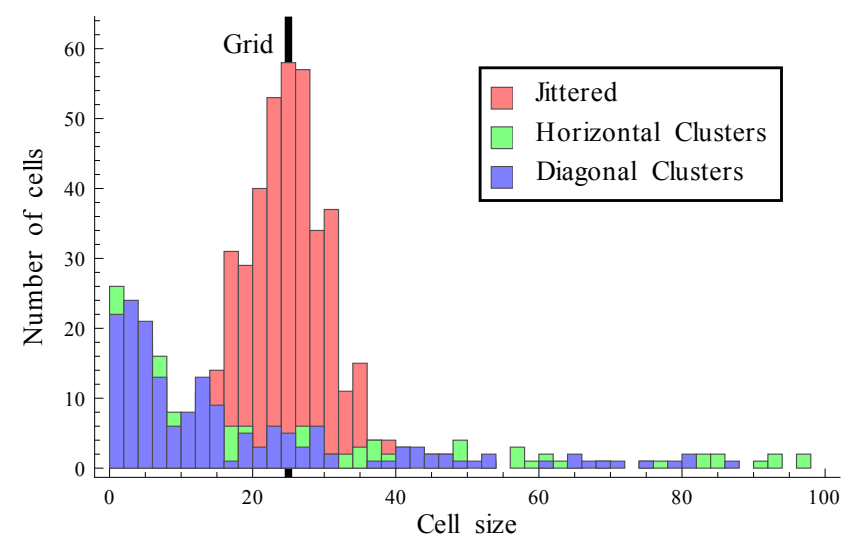

Figure 2. Tile size distribution for jittered and clustered sampling. The jittered sampling is peaked around the grid cell size, with clustered sampling results in many small and few very large tiles. Note that grid and jittered grid have twice as many nodes as the clustered sampling.

the outer regions are poorly sampled, therefore the transition is not visible.

Voronoi tessellation analysis: a Voronoi tessellation assigns a cell to each node, such that every point in the cell is closer to that node than to any other. If the nodes are uniformly distributed in space, the resulting cell sizes will have a clear peak around $A_{\text {sam }} / N_{\text {nod }}$, where $A_{\text {sam }}$ is the size of the total sampled area and $N_{\text {nod }}$ is the number of nodes. However, coarse homogeneous sampling may not be able to make meaningful statements about processes happening on a finer spatial scale. The differences in the sampling can be quantified using the area size distributions of their Voronoi tessellations (Barthélemy, 2011) as shown in Fig. 2.

In the grid all tiles are exactly the same size, so the histogram would be one very sharp peak. In the case of the grid with jitter the peak is broadened. The two clustered distributions peak around much smaller values.

Link length distribution: the link length distribution, shown in row D in Fig. 1, is spiky for the grid and considerably smoother for the jittered node distribution. In clustered sampling, the link length distributions show two peaks. The beige line shows the distance distribution of pairs of points in a continuous square for comparison.

The regular arrangement of points in the grid leads to an overrepresentation of some specific link lengths (i.e. multiples of the grid constant), while other node-node distances are excluded by the node distribution. The distance distribution of pairs of points in a continuous square is the continuous analogue to the link length distribution of the fully connected graph of nodes in the discretized network description. Since climate is a continuous phenomenon, an appropriate discretization should well approximate that distance distribution. The distance distribution is obtained by integrating the distances between all pairs of points $x$ and $y$, which is equivalent to integrating over the area of the square and all

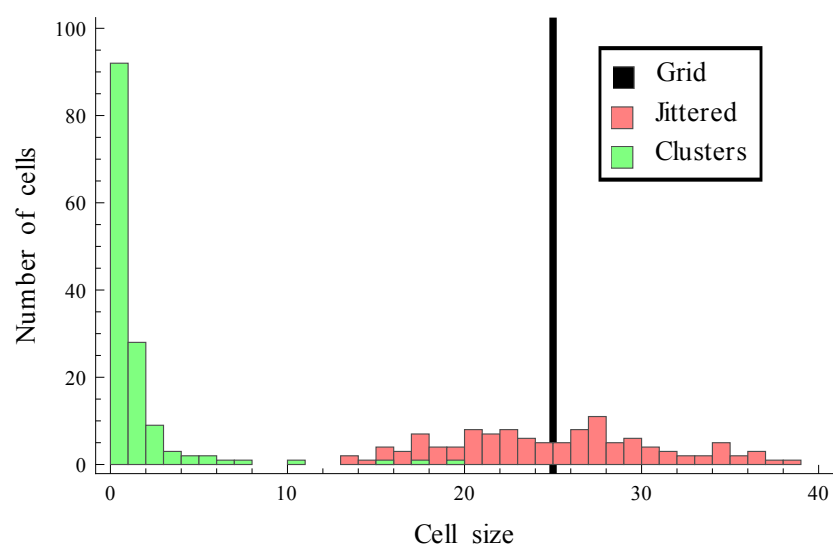

Figure 3. Tile size distribution for the three node distributions in START.

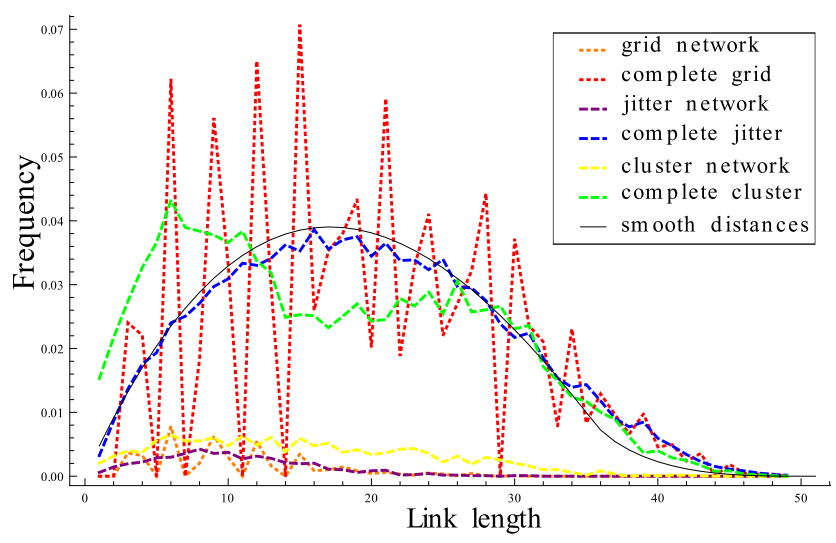

Figure 4. Link length distribution for the three node distributions in START.

circle segments that lie in the square of circles of radius $d$. Normalizing the resulting function of $d$ one gets the distance distribution. It coincides well with the link length distribution of the fully connected nodes of the jittered sampling. The fully connected grids' link length distribution is more spiky but varies around the same curve, while the clustered node sampling leads to a biased link length distribution with one maximum for links within each cluster and another maximum of links connecting the clusters.

Compared to the link length distributions of the fully connected networks, the actual flow networks only express a subset of links that is dominated by short ranged links. In the clustered case Fig. 1Dc this eliminates the inter-cluster peak, because the clusters are not connected by a flow. In the clustered case Fig. 1Dd however, this second peak persists, suggesting that links of length 30 are preferred, even though this effect is entirely a sampling effect and not a property of the flow.

Degree distribution: furthermore we find (results not shown) that the spatial sampling has little impact on the degree distribution. For all sampling types the degree 

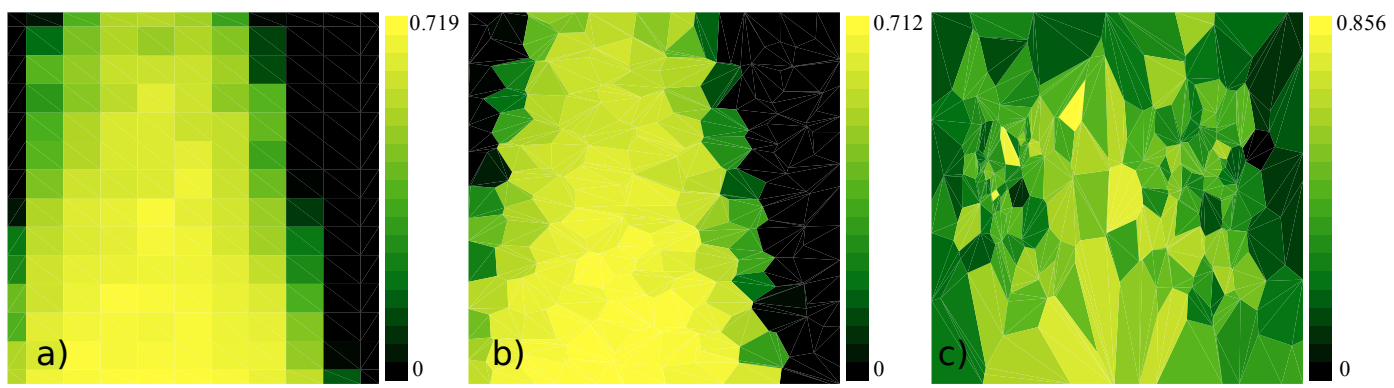

Figure 5. Degree of the network of the flow generated with START for grid (a), grid with jitter (b) and two clusters (c).

distribution was constant with one peak, the position of which depended on the value of the threshold.

Link number with threshold: the total number of links is a decreasing function of the threshold (results not shown). For both, grid and grid with jitter, this function decreases smoothly rather than in steps.

\section{Spatial sampling in networks from the START model}

Next we consider gridded, jittered and clustered networks using the START (Stream Transported Auto Regressive Temperature) model described in Rehfeld et al. (2013).

It is used to simulate time series at each node and construct the network by computing the correlations for each pair. The $20 \%$ strongest correlations are used to set the links in the network. The START model assumes three sources of random information that are transported by three independent flows. The ADE is used to compute variance factors $f_{X}(\boldsymbol{p}, F)$ that approximate the influence of source $X$ on position $\boldsymbol{p}$ at forcing $F$. At each point, the signal is computed as the sum of the contributions from the three sources, scaled with the corresponding variance factor. A local noise contribution is also added:

$$
R_{i}=f_{X}(i, F) R_{X}+f_{Y}(i, F) R_{Y}+f_{Z}(i, F) R_{Z}+R_{\text {noise }} .
$$

Here we use a forcing where only one of the flows is active for better comparison to the diagonal flow in the flow networks.

Despite being also constructed according to advection and diffusion, START is significantly different from the flow networks. Firstly, instead of averaging over all possible initial peak positions, there is a particular source location. This results in a decay of signal strength with increasing distance from the source.

Figure 5 shows the node degrees for the resulting networks, which drop off with distance from the source, unlike the flow networks, where the degree only depended on the velocity. We can see that, while the jittered node distribution shows the same structure as the grid, using clustered node locations leads to a distortion of the pattern.

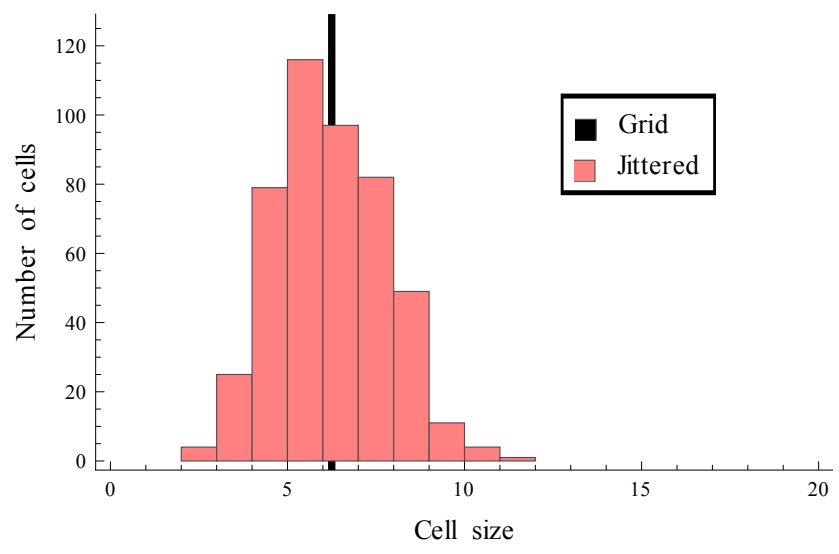

Figure 6. Tile size distribution for the jittered tile size distribution of the reanalysis data together with the grid line.

Additionally we can see that due to the addition of random fluctuations at each point, the degree in the stagnant and faraway regions goes to zero.

The link length distribution is similar to the link length distributions for the flow networks (see Fig. 4). The Tile size distribution (see Fig. 3) shows, that grid and jittered node distribution are homogeneous, while the clusters are not. The betweenness (results not shown) shows the same tongue-like structure as the degree. This can be explained with the random fluctuations reducing the dynamic areas to only one, which is the region inside the flow, while everything else is essentially unconnected.

\section{Spatial sampling effects in data from the Asian monsoon domain}

The data analysis was done on NCEP/NCAR (Kalnay et al., 1996; NCEP/NCAR, 2014) reanalysis daily surface temperature anomalies for the summer monsoon months (June to September) for the years 1971-2010 with a resolution of $2.5^{\circ} \times 2.5^{\circ}$, which results in a grid constant $a=280 \mathrm{~km}$. The network was constructed from the anomaly time series using a Pearson correlation. We threshold the correlation matrix using a link density of the $5 \%$ strongest correlations and obtain 

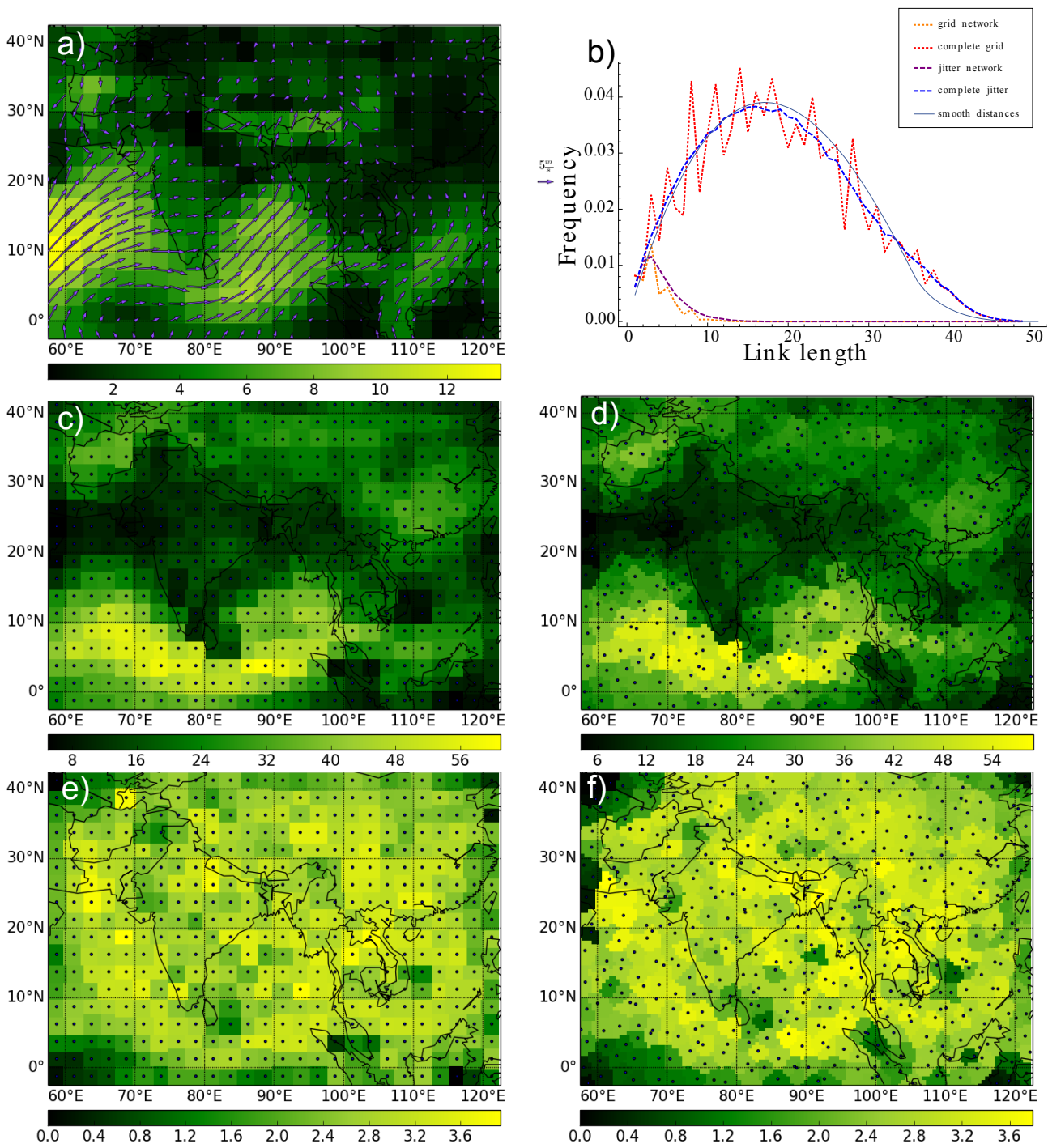

Figure 7. The correlation network of temperature data from NCEP/NCAR on a grid and a jittered grid. (a) Absolute mean wind velocity, (b) link length distributions, (c) degree of the grid network, (d) degree of the jittered network, (e) betweenness of the grid network, (f) betweenness of the jittered network.

degree and betweenness as shown in Fig. 7. The geographical coordinates of each node are marked by a point at the center of each cell. To investigate sampling effects, we added a jitter in longitude and latitude at each grid point with a uniformly random number between $\pm a / 2$. The tile size distributions are shown in Fig. 6.

The new time series are constructed as Gaussian weighted averages of all of the original time series on the grid. The weights depend on the euclidean distance between the grid points and the new location.

Figure 7 shows (a) the annual average of the surface wind speeds, where the arrow displays the direction and the color shows the absolute value, which is computed by averaging over longitudinal and latitudinal components separately and taking the absolute value of the resulting vectors. Subfigure (b) shows the link length distribution. The degree is presented for grid and jittered sampling in the second row (c, d). The bottom row shows the betweenness for grid and jittered sampling (e, f).

The node degree (Fig. 7c and d) is highest over the ocean and lowest over the Indian subcontinent. This coincides with the absolute wind velocities, which are highest over the ocean, Bangladesh and the Himalayas. This supports the theory that connections in the climate network are often caused by an atmospheric or ocean flow between the node locations. On a daily scale, temperature fluctuations are transported along the flow, leading to a high correlation of nodes connected by a high velocity. Despite the high wind speeds over 
the Himalayas, however, the degrees there are not elevated. This may be because the plateau is relatively secluded from the rest of the network due to its high altitude. Consequently, even if the link density inside the secluded area were very high, the degree would be low, as connections to other regions are less likely.

We find that the effect of spatial sampling on the degree patterns is small and the overall patterns are not affected by the node distribution. This coincides well with our findings from the theoretical approaches in Sects. 2 and 3, as the jittered node distribution is still homogeneous as shown in the Voronoi tile size distribution in Fig. 6.

The shortest-path betweenness $(\mathrm{e}, \mathrm{f})$ is more sensitive to the spatial distribution of the nodes and the spatial resolution. Therefore, few clear patterns can be seen, and most regions have a betweenness between 2.5 and 3.5. The jittered network shows higher boundary effects in the west. There are three regions with a particularly low betweenness in both the gridded and the jittered version of the network at (80$\left.85^{\circ} \mathrm{E}, 5-10^{\circ} \mathrm{N}\right),\left(100^{\circ} \mathrm{E}, 0-5^{\circ} \mathrm{N}\right)$ and $\left(110^{\circ} \mathrm{E}, 10-15^{\circ} \mathrm{N}\right)$, these coincide with small regions with a low degree.

\section{Discussion and conclusions}

We have analyzed the influence of the spatial distribution of nodes on the network topology for three examples: flow networks, the START model and surface temperature correlation networks. We find that the degree is robust, while the betweenness reacts more sensitively to the spatial sampling. All these examples are related to climate, yet the results can be generalized for all networks coming from discretizations of any continuous system.

For a smooth link length distribution, the node-to-node separation has to cover all possible distances. A grid does not fulfill that, as all distances are of the form $d\left(x_{1}, x_{2}\right)=a \sqrt{n^{2}+m^{2}}$, where $a$ is the grid constant and $n$ and $m$ are integer numbers. Distances that can not be represented in this way are not present in a grid.

While the effects of the area sizes themselves are discussed in detail in Heitzig et al. (2012) and can be removed using their proposed consistently weighted network measures, this study shows that the problem goes further, when considering the underlying physical system. While the node size distribution in the two clustered sampling versions discussed in Sect. 2 is very similar, their relative position to the underlying flow is not, and they have very different distorting effects on the network structure.

In future work it might be interesting to compare different jitter realizations quantitatively, for example using the common component evolution function (CCEF) as introduced in Tupikina et al. (2013).

So in summary, we found that, as long as the node distribution in space is sufficiently homogeneous, the exact spatial sampling chosen has little impact on the topology of the network in all cases we analyzed. Only in cases of significantly inhomogeneous sampling, distortion and misleading structures arose. It is therefore important to discuss the sampling and its impact when analyzing spatially sampled data. As a simple test of the spatial sampling we suggest looking at the Voronoi size distribution. In a homogeneous sampling this will have a clear peak around $A_{\text {sam }} / N_{\text {nod }}$, where $A_{\text {sam }}$ is the size of the total sampled area and $N_{\text {nod }}$ is the number of nodes. If the peak is shifted or very spread out, that suggests poor spatial sampling.

Acknowledgements. The authors acknowledge funding by the German Science Foundation (DFG graduate school 1539), the German Federal Ministry for Education and Research (BMBF project PROGRESS, support code 03IS2191B) and the support of the LINC project (No. 289447) funded by EC's Marie-Curie ITN program (FP7-PEOPLE-2011-ITN). K. Rehfeld was supported by the Helmholtz grant VG-900NH. We would like to thank Norbert Marwan for discussion.

Edited by: M. Small

Reviewed by: three anonymous referees

\section{References}

Barthélemy, M.: Spatial networks, Phys. Rep., 499, 1-101, doi:10.1016/j.physrep.2010.11.002, 2011.

Boccaletti, S., Latora, V., Moreno, Y., Chavez, M., and Hwang, D.: Complex networks: Structure and dynamics, Phys. Rep., 424, 175-308, doi:10.1016/j.physrep.2005.10.009, 2006.

Dippé, M. and Wold, E.: Antialiasing through stochastic sampling, ACM Siggraph Computer Graphics, 19, 69-78, 1985.

Donges, J. F., Zou, Y., Marwan, N., and Kurths, J.: Complex networks in climate dynamics, Eur. Phys. J. Spec. Top., 174, 157179, doi:10.1140/epjst/e2009-01098-2, 2009.

Heitzig, J., Donges, J., Zou, Y., Marwan, N., and Kurths, J: Nodeweighted measures for complex networks with spatially embedded, sampled, or differently sized nodes, Eur. Phys. J. B, 85, 121, doi:10.1140/epjb/e2011-20678-7, 2012.

Hlinka, J., Hartman, D., Vejmelka, M., Novotná, D., and Palùs, M.: Non-linear dependence and teleconnections in climate data: sources, relevance, nonstationarity, Clim. Dynam., doi:10.1007/s00382-013-1780-2, in press, 2013.

Kalnay, E., Kanamitsu, M., Kistler, R., Collins, W., Deaven, D., Gandin, L., Iredell, M., Saha, S., White, G., Woollen, J., Zhu, Y., Chelliah, M., Ebisuzaki, W., Higgins, W., Janowiak, J., Mo, K. C., Ropelewski, C., Wang, J., Leetmaa, A., Reynolds, R., Jenne, R., and Joseph, D.: The NCEP/NCAR 40-year reanalysis project, B. Am. Meteorol. Soc., 77, 437-440, 1996.

Kosmidis, K., Havlin, S., and Bunde, A.: Structural properties of spatially embedded networks, Europhys. Lett., 488, 129, doi:10.1038/488129b, 2008.

Malik, N., Bookhagen, B., Marwan, N., and Kurths, J.: Analysis of spatial and temporal extreme monsoonal rainfall over South Asia using complex networks, Clim. Dynam., 39, 971987, doi:10.1007/s00382-011-1156-4, 2011. 
Molkenthin, N., Rehfeld, K., Marwan, N., and Kurths, J.: Networks from flows - from dynamics to topology, Sci. Reports, doi:10.1038/srep04119, in press, 2013.

NCEP/NCAR: NCEP/NCAR, http://www.erls.noaa.gov/psd, last access: February 2014.

Rehfeld, K., Marwan, N., Heitzig, J., and Kurths, J.: Comparison of correlation analysis techniques for irregularly sampled time series, Nonlin. Processes Geophys., 18, 389-404, doi:10.5194/npg18-389-2011, 2011.

Rehfeld, K., Marwan, N., Breitenbach, S. F. M., and Kurths, J.: Late Holocene Asian summer monsoon dynamics from small but complex networks of paleoclimate data, Clim. Dynam., 41, 3-19, doi:10.1007/s00382-012-1448-3, 2012.

Rehfeld, K., Molkenthin, N., and Kurths, J.: Spatio-temporal climate transitions from paleoclimate networks, Nonlin. Process Geophys., accepted, 2013.

Rheinwalt, A., Marwan, N., Kurths, J., Werner, P., and Gerstengarbe, F.-W.: Boundary effects in network measures of spatially embedded networks, Europhys. Lett., 100, 28002, doi:10.1209/0295-5075/100/28002, 2012.
Stolbova, V., Martin, P., Bookhagen, B., Marwan, N., and Kurths, J.: Topology and seasonal evolution of the network of extreme precipitation over the Indian subcontinent and Sri Lanka, Nonlin. Process Geophys., in review, 2014.

Tsonis, A. A., Swanson, K. L., and Roebber, P. J.: What Do Networks Have to Do with Climate?, B. Am. Meteorol. Soc., 87, 585-595, doi:10.1175/BAMS-87-5-585, 2006.

Tsonis, A. A., Wang, G., Swanson, K. L., Rodrigues, F. A., and Costa, L. D. F.: Community structure and dynamics in climate networks, Clim. Dynam., 37, 933-940, doi:10.1007/s00382-0100874-3, 2010.

Tupikina, L., Rehfeld, K., Molkenthin, N., Stolbova, V., Marwan, N., and Kurths, J.: Detecting evolution of networks using spatialtemporal autocorrelation function, Nonlin. Process Geophys., accepted, 2013.

Unser, M.: Sampling-50 years after Shannon, Proc. IEEE, 88, 569587, doi:10.1109/5.843002, 2000.

Yamasaki, K., Gozolchiani, A., and Havlin, S.: Climate Networks around the Globe are Significantly Affected by El Niño, Phys. Rev. Lett., 100, 228501, doi:10.1103/PhysRevLett.100.228501, 2008. 